\title{
Stereotyped Movements Among Children Who Are Visually Impaired
}

\section{Eynat Gal and Murray J. Dyck}

Abstract: Does the severity of visual impairment affect the prevalence and severity of stereotyped movements? In this study, children who were blind or had low vision, half of whom had intellectual disabilities, were assessed. The results revealed that blindness and global delays were associated with more sensory processing dysfunction and more stereotyped movements.

Stereotyped movements-motor responses that are repetitious and excessive in rate, frequency, or amplitude and are pursued in an invariant way-are a defining characteristic of autism (American Psychiatric Association, 2000; Schopler, 1995), but are also common among children with visual impairments, that is, those who are blind or have low vision (Tröster, Brambring, \& Beelmann, 1991) and those with other disabilities (Murdoch, 1996; Rojahn \& Sisson, 1990). When stereotyped movements cannot be accounted for as a compulsion, tic, or part of a pervasive developmental disorder, they are the essential feature of a stereotypic movement disorder if they interfere with normal activities or result in selfinjury (American Psychiatric Association, 2000). In addition to being perceived as inappropriate, odd, and lacking adaptive significance (Baumeister, 1978; Turner, 1999), self-injurious stereotyped movements are clearly maladaptive be-

This study was supported by Australian Postgraduate Awards to the first author. Thanks are expressed to the teachers and students at various schools in the north of Israel who took part in the study. cause they can cause severe damage to, and even threaten the lives of, the children who perform them. Self-injurious stereotyped movements are rarely evident among typical children, but have been reported in $52 \%$ of children with visual impairments (Gal, Dyck, \& Passmore, in press).

Some stereotyped movements, such as eye poking and pressuring the eyeball, appear to be relatively specific to children with visual impairments, especially those with an intact optic nerve but a damaged cornea (Tröster et al., 1991). Other stereotyped movements, although not specific to visual impairment, appear to be related to a specific cause of visual impairment when a child is blind or has low vision. Rocking, for example, appears to be strongly associated with retinopathy of prematurity (Jan, Freeman, \& Scott, 1977; McHugh \& Pyfer, 1999; McHugh \& Lieberman, 2003). Although there is some evidence that stereotyped movements are related to the severity and age of onset of the visual impairment (Jan et al., 1977), how different forms of stereotyped movements vary as a function of the severity of the visual impairment is 
not known. There is reason to believe that children who are blind perform more stereotyped movements, including selfinjurious ones, than children with low vision.

It has been proposed that stereotyped movements are, in fact, adaptive because they help under- or overstimulated children to maintain an optimal or homeostatic state of stimulation (Miller, Lane, Cermak, Anzalone, \& Osten, 2005). Children who are blind can be expected to be understimulated compared to those with some vision. In addition, total or near-total vision loss may lead to a more generalized dysfunction in sensory processing as a partial consequence of the absence of visual stimulation (Gal, 2006). Sensory processing refers to the way in which the nervous system manages sensory information, including the registration, modulation, integration, and organization of sensory input (Miller \& Lane, 2000).

Miller et al. (2005) identified three types of sensory processing disorders. In sensory modulation disorder, a person has difficulty regulating and organizing the type and intensity of his or her responses to sensory input from the body (such as movement) or the environment (such as sound) (Miller, Anzalone, Lane, Cermak, \& Osten, 2007). In sensory discrimination disorder, a person has difficulty processing the spatial and temporal qualities of touch, movement, or body position; vision; and audition, all of which are important for the development of skills (Koomar \& Bundy, 2002). In sensorybased motor disorder, the difficulty is with posture and motor planning and is often shown by poor tactile and proprioceptive processing; clumsiness; poor gross motor, fine motor, and manipulation skills; and poor organization. Children with sensory-based motor disorders may have difficulties with balance, sequencing movements, bilateral coordination, and imitating movements (Miller et al., 2005; Smith \& Bryson, 1994). Such difficulties are especially pronounced when the children learn a new motor skill, which requires more practice for them than it does for typical children to automate the new skill.

If a child experiences severe understimulation in one modality, as in the case of visual impairment, or either severe underor overstimulation in intense sensory processing dysfunction, the function of stereotyped movements would be to increase stimulation in an understimulating environment (Mason, 1991) or to block excessive stimulation associated with sensory processing dysfunction (Wehmeyer, 1989); a change in the level of stimulation would help a person to remain within optimal physiological or psychological limits (Fraser \& Broom, 1990).

Stereotyped movements may be exacerbated by the presence of a visual impairment because of the relationship between the visual impairment and motor development. A visual impairment adversely influences motor development and the ability to integrate information from other sensory modalities (Dyck, Farrugia, Shochet, \& Holmes-Brown, 2004; Gosch, Brambring, Gennat, \& Rohlmann, 1997; Jan, Groenveld, \& Connolly, 1990; Tröster \& Brambring, 1993). Children with visual impairments have fewer opportunities for vigorous physical activity at school or at home. They regularly miss physical education classes and recess to receive specialized services, such as braille instruction, and when they do attend these activities, the time they devote 
to vigorous movement is often brief (McHugh \& Lieberman, 2003). Safety concerns; families' and teachers' lack of knowledge about motor development; and the cognitive, physical, and socialemotional consequences of blindness all limit a child's exposure to the experiences that are necessary to acquire motor planning and performance skills (Gosch et al., 1997; Jan et al., 1990; O'Donnell \& Livingston, 1991; Tröster \& Brambring, 1993; Tröster et al., 1991). Problems of motor development may be a cause of internal disequilibrium that is independent of sensory processing dysfunction and may increase the need for a child to engage in stereotyped movements to regain homeostasis.

There is some evidence that stereotyped movements are related to sensory processing dysfunction and to intelligence (Gal, Dyck, \& Passmore, in press). However, it is not known whether sensory loss is related to stereotyped movements independently of sensory processing dysfunction or intelligence or whether the nature and degree of sensory impairment affects the location and amount of stereotyped movements. The aim of this study, therefore, was to assess whether children who are blind perform more numerous, more severe, and different stereotyped movements than do children with low vision. Other aims were to assess whether any such effects are mediated by the level of sensory processing dysfunction and whether any such effects are independent of a child's intellectual ability.

\section{Method}

\section{Participants}

Participants were recruited following approval of this project by the Human Re- search Ethics Committee of Curtin University of Technology, Perth, Australia. The participants were 50 children (25 boys and 25 girls) aged 6 to 13 (mean age $=9.02, S D=1.59$ ). All the participants lived with their families and attended school in northern Israel. The sample included 25 children with and 25 children without intellectual disabilities. Children with intellectual disabilities met the DSM-IV criteria for a diagnosis of mental retardation [intelligence quotient (IQ) and adaptive functioning index of less than 70; American Psychiatric Association, 2000]. These children were recruited from schools that specialized in educating children with special needs or from specialist classes in regular state schools. The children without intellectual disabilities were recruited from specialized after-school programs for children with visual impairments. All the children had been defined by medical services as having a visual impairment that was great enough for them to be eligible for special educational support.

The children were divided into two groups on the basis of the severity of the visual impairment. The group who were blind consisted of 21 children, 11 of whom were intellectually disabled. Children in this group lacked form and visual light perception or had residual vision that was sufficient only to distinguish darkness from light. These children all depended on their other senses and adaptive devices to function in daily life. The group of children who had low vision consisted of 29 children with somewhat greater residual vision, 14 of whom were intellectually disabled. Children in this group had a visual acuity of less than $6 / 60$ or a visual field of 20 degrees or less. 
These children also depended on their other senses and adaptive devices to function in daily life. The two groups did not differ in age or gender.

Children were excluded if they had been diagnosed with specific syndromes that are closely associated with specific stereotyped movements, including Lech Nyhan syndrome, Cornelia de Lange syndrome, Rilez Day familial dysautonomia, fragile X syndrome, and Rett syndrome. These syndromes are associated with an abnormal metabolism or a specific $\mathrm{x}$-linked gene (or both) and have known sensory abnormalities that distinguish them from other populations with intellectual disabilities (see American Psychiatric Association, 2000, pp. 131-132). Children with tardive dyskinesia and children who had a dual diagnosis of autism and visual impairment were also excluded.

\section{Measures}

The Short Sensory Profile (McIntosh, Miller, Shyu, \& Dunn, 1999) is a Likertstyle rating scale for parents or teachers that measures sensory processing difficulties in children. Although it is typically used with children aged 5-10, in the context of persons with disabilities the profile is used with persons up to age 21 years (Dunn, 1999). The Short Sensory Profile was designed for screening and research use and contains 38 of the 125 items in the original Sensory Profile that best discriminate children with and without sensory processing disorders. It has seven sections: tactile sensitivity, taste or smell sensitivity, movement sensitivity, underresponsive or seeks sensation, auditory filtering, low energy or weak sensitivity, and visual or auditory sensitivity. Item scores within each section are summed to yield a section score, and section scores are summed to yield a total summary score. Section scores are categorized within three ranges: "typical performance," "probable difference," and "definite difference." These categories are based on a classification system derived from norms, that is, the range of scores of children without disabilities as they performed on each subtest. This system allows the construction of individual profiles, comparing each child's performance to normative performance. The test has been found to identify sensory difficulties among children with a sensory modulation disorder (Miller, Reisman, McIntosh, \& Simon, 2001), autism and fragile $X$ syndrome (Miller et al., 2001; Rogers, Hepburn \& Wehner, 2003), and attention deficit disorder (Mangeot et al., 2001). To our knowledge, the Short Sensory Profile has not been used in previous research with children who are visually impaired, and so its reliability (coefficient alpha) in this population was assessed. The results indicated acceptable internal consistency for the total summary score (alpha $=.84$ ) and for the section scores (tactile sensitivity, alpha $=.78$; taste or smell sensitivity, alpha $=.79$; movement sensitivity, alpha $=.91$; underresponsive or seeks sensation, alpha $=.82$; auditory filtering, alpha $=.75$; low energy or weak sensitivity, alpha $=.88$; and visual or auditory sensitivity, alpha $=.63$ ).

The Stereotyped and Self-Injurious Movement Interview is a 32-item clinicianadministered questionnaire that is designed to assess 10 kinds of stereotyped body movements (for example, pacing, spinning, and rocking), 5 kinds of stereotyped manipulation of objects (including operating switches, arranging objects, or mouthing or 
sucking objects), and 10 kinds of stereotyped self-injurious movements (such as hitting one's head, biting one's hands, and picking or scratching one's body cavities) in typically developing children and children with visual or auditory impairments or a developmental disorder or both (Gal, Dyck, \& Passmore, 2002; Gal, Dyck, \& Passmore, 2009). With this interview, raters are asked to indicate if each form of movement has occurred at least once a week for at least three months. Only movements that occur with at least this frequency are scored as present. Among other things, the interview assesses how many different kinds of stereotyped movements a child performs, so that scores may range from 0 to 5 for the stereotyped manipulation of objects (all forms of object manipulation are present) and from 0 to 10 for stereotyped body movements and for stereotyped selfinjurious behaviors (see Gal et al., 2009, for a complete list of stereotyped movements). The interview is sensitive to differences between children who are visually impaired and children with other sensory disorders (such as deafness) or developmental disorders (like autism) in the frequency or severity of stereotyped movements (Gal et al., 2009). The reliability of the scale for all the populations for which the interview was designed is alpha $=.79$ (Gal et al., in press), but is necessarily lower when used with specific populations, like children who are visually impaired (alpha $=.54$ ), who differ from each other as to which stereotyped movements are performed (Gal et al., 2009).

\section{Procedure}

The Short Sensory Profile and Stereotyped and Self-Injurious Movement Interview were administered to the partici- pants' teachers by the first author as a face-to-face or telephone interview in their home schools. Teachers' ratings were preferred to direct observation to enhance the feasibility of the research, but also because they were expected to provide more reliable information, especially for low-frequency stereotyped movements (1-5 times per week), which may not be performed during standard observation periods. Teachers' ratings are commonly used to assess a broad range of problem behaviors, including inattention, impulsivity, and hyperactivity (Gomez, 2008); depression, anxiety, and aggression (Epkins, 1996); academic skills (Foegen, 2008); learning potential (Hamers, Hessels, \& Pennings, 1996); and language ability (see, for example, Massa, Gomes, Tartter, Wolfson, \& Halperin, 2008), among other behaviors (Gadow, Sprafkin, Salisbury, Schneider, \& Loney, 2004). Interviews lasted approximately 60 minutes per child.

The teachers knew the children well. For children with intellectual disabilities, the raters were special education teachers in specialized schools, who were trained and experienced in working with children with visual impairments and provided daily special support, in addition to that provided by the classroom teacher, on a one-to-one basis, throughout the school day, in the context of small classes of six to eight children. For children without intellectual disabilities, the raters were also special education teachers who provided several hours of daily support, also on a one-to-one basis, in an afterschool program for children with special needs. 


\section{Table 1}

Prevalence of different categories of stereotyped movement by severity of vision loss and intellectual level.

\begin{tabular}{|c|c|c|c|c|c|c|c|c|c|}
\hline \multirow[b]{2}{*}{ Visual status } & \multirow[b]{2}{*}{$\mathbb{I Q}$} & \multicolumn{2}{|c|}{ SM objects } & \multicolumn{2}{|c|}{ SM body } & \multicolumn{2}{|c|}{ SI SM } & \multicolumn{2}{|c|}{ SSP } \\
\hline & & Mean & $S D$ & Mean & $S D$ & Mean & SD & Mean & $S D$ \\
\hline \multirow[t]{2}{*}{ Low vision } & $>69$ & .46 & .74 & 1.20 & 1.61 & .13 & .35 & 162.47 & 17.25 \\
\hline & $<70$ & 1.92 & .73 & 1.71 & 1.26 & .71 & .82 & 139.79 & 6.91 \\
\hline \multirow[t]{2}{*}{ Blind } & $>69$ & .60 & .69 & 2.20 & 1.61 & .70 & .67 & 148.90 & 14.59 \\
\hline & $>70$ & 1.27 & 1.27 & 2.72 & 1.67 & 1.54 & .68 & 136.73 & 15.68 \\
\hline
\end{tabular}

Note: SM objects = stereotyped manipulation of objects; SM body = Stereotyped body movements; SI $\mathrm{SM}=$ self-injurious stereotyped movements; and SSP = Short Sensory Profile summary score.

\section{Results}

Descriptive statistics (means and standard deviations) for the prevalence of stereotyped movements and summary scores on the Short Sensory Profile are reported in Table 1 . All forms of stereotyped movement were more common in the group of children who were blind and in the subgroups of children who were intellectually disabled. The identical pattern was evident in the scores on the Short Sensory Profile, with more sensory processing dysfunction (lower scores) evident in the group of children who were blind and in the subgroups of children who were intellectually disabled.

The analysis began with a two (severity of visual impairment) by two (intellectual level) analysis of variance of summary scores on the Short Sensory Profile to assess whether the groups differed in the severity of sensory processing dysfunction. The results indicated significant effects for visual impairment $[F(1$, 46) $=4.21, p<.05$, epsilon squared $=$ $.08]$ and for intellectual level $[F(1$, $46)=18.52, p<.001$, epsilon squared $=.28]$, but not for the interaction. Pearson correlations were calculated to assess the strength of any linear relationship between sensory processing dys- function and stereotyped movements within and across the samples. The results (see Table 2) indicated significant and sometimes strong relationships between the Short Sensory Profile summary score and one or more forms of stereotyped movements in each sample and in the combined sample. Sensory processing dysfunctions were consistently related to the stereotyped manipulation of objects and were usually related to stereotyped self-injurious movements, but not to stereotyped body movements. For this reason, the summary

\section{Table 2}

Correlations between summary scores on the Short Sensory Profile and categories of stereotyped movements.

\begin{tabular}{lccc}
\hline & \multicolumn{3}{c}{ Stereotyped movements } \\
\cline { 2 - 4 } Visual status & Body & Objects & Injurious \\
\hline Combined sample & & & \\
Summary & -.26 & $-.57^{\star \star}$ & $-.40^{\star \star}$ \\
Body & & .18 & $.52^{\star \star}$ \\
Objects & & & $.37^{\star *}$ \\
Low vision & -.30 & $-.70^{\star *}$ & $-.36^{\star}$ \\
Body & & .25 & $.38^{\star}$ \\
Objects & & & $.50^{* *}$ \\
Blind & & & \\
Summary & -.05 & $-.50^{\star}$ & -.30 \\
Body & & .20 & $.52^{\star}$ \\
Objects & & & $.48^{\star}$ \\
\hline
\end{tabular}

* significant at the .05 level (two-tailed); ** significant at the .01 level (two-tailed). 
scores on the Short Sensory Profile were used as a covariate in subsequent betweengroup comparisons.

A two (severity of visual impairment) by two (level of intelligence) multivariate analysis of covariance of the set of stereotyped movement scores (body movements, manipulation of objects, and selfinjurious behavior) revealed a significant effect for the covariate $[F(3,43)=3.24$, $p<.05$, epsilon squared $=.18]$ and significant main effects for the extent of visual impairment $[F(3,43)=7.18, p=$ .001 , epsilon squared $=.33]$ and intellectual level $[F(3,43)=4.22, p<.05$, epsilon squared $=.22$, but not for the interaction. Univariate tests indicated that, consistent with the results shown in Table 2, the covariate was significant only for the stereotyped manipulation of objects $[F(1,45)=9.87, p<.01$, epsilon squared $=.18]$. The main effect for the severity of visual impairment was significant for self-injurious behavior $[F(1$, $45)=11.17, p<.01$, epsilon squared $=$ $.19]$ and the manipulation of objects $[F(1$, $45)=4.82, p<.05$, epsilon squared $=$ .09], but only marginally significant for body movements $[F(1,45)=3.70, p=$ .061 , epsilon squared $=.07]$. Similarly, the main effect for intellectual level was significant for self-injurious behavior $[F(1,45)=7.76, p<.01$, epsilon squared $=.14]$ and the manipulation of objects $[F(1,45)=8.69, p<.01$, epsilon squared $=.16]$, but not for body movements.

Finally, follow-up chi-square analyses of individual stereotyped movement items were conducted to assess which specific forms of behavior differed for the children who were blind and the children with low vision. Significant between- group differences were observed for six items: staring, which was more common in the group of children with low vision, and rocking, head or neck movements, head banging, eye gouging, and picking at body cavities, which were all more common in the group of children who were blind. These group differences in self-injurious behaviors contributed to the finding that some $81 \%$ of the children who were blind, compared with just $31 \%$ of the children with low vision, performed at least one form of self-injurious behavior. In terms of intellectual level, significant differences were found for eight items-object manipulation, operating switches, sucking objects, touching body parts or clothing, making noises, head banging, hitting one's head or other body parts, and throwing oneself against the wall or floor-which were all more common in the subgroup of children who were intellectually disabled.

\section{Discussion}

The first aim of the study presented here was to examine whether and how children who are blind differ from those with low vision in their stereotyped movements, including self-injurious behaviors. The results indicated that the group who were blind engaged in more stereotyped movements, specifically, stereotyped manipulation of objects and self-injurious behaviors. These two groups also differed in terms of the kind and location of body movements. The children who were blind were more likely to engage in some specific body movements, including rocking and head or neck movements, whereas those with low vision were more likely to engage in staring. These results are consistent with previous evidence that 
rocking and eye gouging are the most common stereotyped movements among children who are blind (Brambring \& Tröster, 1992; Tröster et al., 1991) and imply that disability-specific characteristics, such as the kind and severity of sensory loss, shape the kind of stereotyped movements that a child is most likely to perform.

The second aim of this study was to examine whether these differences were mediated by the severity of a sensory processing dysfunction and whether they were independent of a child's level of intellectual functioning. The results indicated that group differences are not mediated by the severity of sensory processing dysfunction. Rather, the severity of sensory processing dysfunction, the severity of visual impairment, and level of intellectual functioning make separate contributions to stereotyped movements.

Researchers have asked whether the higher levels of stereotyped movements in children who are blind are mediated by greater sensory processing dysfunction in these children. The conditions that are responsible for blindness may cause aberrant processing of other forms of sensory stimuli. In addition, blindness adversely influences motor development and the ability to integrate information from other sensory modalities (Dyck et al., 2004; Gosch et al., 1997; Jan et al., 1990; Tröster \& Brambring, 1993). An extended sensory processing dysfunction would make a child experience stimulation from various senses in different ways and would make the child overresponsive, underresponsive, or a sensation seeker (Miller et al., 2005). The stereotyped movements could then serve to block the aversive stimuli and maintain homeosta- sis (Fraser \& Broom, 1990; Wehmeyer, 1989). The results showed that sensory processing dysfunction is related to the increased manipulation of objects at all levels of visual impairment.

The strong relationship between sensory processing dysfunction and some stereotyped movements is consistent with the idea that stereotyped movements are adaptive and allow a child to maintain an optimal level of stimulation. The stereotyped movements may represent a response to massive understimulation and limited alternatives for obtaining appropriate stimulation (Mason, 1991). Also consistent with this idea is the finding that children who are blind and children with low vision engage in different repetitive movements. Each group engages in movements that may provide direct compensation for its sensory loss. For example, children with low vision engage in staring behavior, which may maximize visual stimulation in children whose visual inputs are limited but nonetheless present. For children who are blind, such behavior has no function and so is not performed. Children who are blind are more likely to engage in rocking and repetitive head movements, which provide proprioceptive and vestibular stimulation, which they receive much less of than do sighted children and children with low vision (Gosch et al., 1997; O'Donnell \& Livingston, 1991; Tröster \& Brambring, 1993).

The functions of self-injurious behaviors, such as head banging, eye gouging, and picking at body cavities, which were more common in children who are blind, are not clear. One possibility is that rubbing or pressing the eye may stimulate the optic nerve endings and evoke so-called 
phosphenes, sensations of light at the cortical level (Fazzi et al., 1999). These stereotyped movements are observed mainly in children with peripheral blindness, who, having intact optic nerves, are able to perceive light and dark. A similar explanation may apply to head banging, since the topographical proximity of these movements may suggest a similar function.

The finding that intellectual level and severity of visual impairment made separate contributions to stereotyped movements is consistent with research that has shown that children with intellectual disabilities, but no sensory disturbance, perform more stereotyped movements than do typical children (Rojahn \& Sisson, 1990). However, although the intellectual level makes a separate contribution to the number of stereotyped movements that are performed, the results did not show an interaction between visual impairments and intellectual level. In fact, of the six stereotyped movements that distinguished children who were blind from children with low vision, only one (head banging) overlapped with the eight stereotyped movements that distinguished children with and without intellectual disabilities. This lack of interaction is surprising because other research has suggested that an intellectual disability amplifies the effects of sensory loss (Murdoch, 1996; Poustka \& Lisch, 1993). The results of our study suggest that the severity of blindness has effects over and above such variables as intellectual level or general sensory processing. They indicate that the severity of a sensory loss has additive consequences in terms of how many and which stereotyped movements a child performs, and they therefore reinforce the importance of assessing how each disability and each combination of disabilities contributes to stereotyped movements.

\section{Conclusions, limitations, and recommendations}

Although each disability is linked to a specific pattern of stereotyped movements, the function of the movements is presumably the same in all cases: to help a person maintain an optimal level of internal stimulation. Different disabilities limit or distort sensory systems in different ways and so require a person to engage in different forms of compensatory behavior. The practical challenge is to identify the function of stereotyped movements for a specific child-Is it to compensate for under- or overstimulation and in which sensory systems?-and to help the child acquire a repertoire of behavior that achieves the same function while being more adaptive in other ways.

The limitations of this research have to be acknowledged. Our use of teachers' ratings of stereotyped movements means that our behavioral sampling was limited to the period when the children were in school or receiving special education services, so it cannot be ascertained that the stereotyped movements that occurred during this period were representative of the children's movements in general. However, at present, there is no reason to doubt that the same relationships between stereotyped movements and sensory processing or intelligence or the severity of visual impairments would be evident for these movements performed at other times. Since we had no access to the children's IQ scores, but only to categorical data about the children's intellectual levels, we could not directly assess the relationships between intelligence and 
sensory processing disturbances or stereotyped movements. Finally, our samples were not only small, but were drawn from a narrow geographic and social region, and pending replication, we cannot assume that these results will generalize to other groups of children who are blind and children who have low vision.

Further research on functional relationships between sensory and motor systems, specifically on the additive and interactive effects of specific sets of sensory losses and impairments on repetitive movements, is necessary to meet the practical challenge of helping children who are visually impaired and other children with disabilities to respond more adaptively to over- or understimulation. It is also important to assess how functional relationships between sensory and motor systems interact with other systems that are functionally related to stereotyped movements, including poor functional communication skills (see Kurtz et al., 2003).

Further research should include information on each child's adaptive functioning, functional communication skills, or other characteristics that may be functionally related to stereotyped movements. It is important to examine whether such characteristics moderate the relationship between sensory processing and stereotyped movements in children who are blind versus those with low vision. Understanding these relationships may assist professionals to tailor interventions to the needs of individual children with visual impairments.

\section{References}

American Psychiatric Association. (2000). Diagnostic and statistical manual of mental disorders (4th ed.). Washington, DC: Author.
Baumeister, A. A. (1978). Origins and control of stereotyped movements. In C. E. Meyers (Ed.), Quality of life in severely and profoundly mentally retarded people: Research foundations for improvement (Monograph No. 3). Washington, DC: American Association for Mental Deficiency.

Brambring, M., \& Tröster, H. (1992). On the stability of stereotyped behaviors in blind infants and preschoolers. Journal of Visual Impairment \& Blindness, 35, 775-781.

Dunn, W. (1999). Sensory Profile: User's manual. San Antonio, TX: Psychological Corporation.

Dyck, M., Farrugia, C., Shochet, I., \& HolmesBrown, M. (2004). Emotion recognition/ understanding ability in hearing or visionimpaired children: Do sounds, sights, or words make the difference? Journal of Child Psychology \& Psychiatry, 45, 789-800.

Epkins, C. C. (1996). Parent ratings of children's depression, anxiety, and aggression: A cross-sample analysis of agreement and differences with child and teacher ratings. Journal of Clinical Psychology, 52, 599-608.

Fazzi, E., Lanners, J., Danova, S., FerrarriGinevra, O., Gheza, C., Luparia, A., Umberto Balottin, U., \& Lanzi, G. (1999). Stereotyped behaviors in blind children. Brain Development, 21, 522-528.

Foegen, A. (2008). Progress monitoring in middle school mathematics: Options and issues. Remedial and Special Education, 29, 195-207.

Fraser, A. F., \& Broom, D. M. (1990). Farm animal behaviour and welfare. London: Bailliere Tindall.

Gadow, K. D., Sprafkin, J., Salisbury, H., Schneider, J., \& Loney, J. (2004). Further validity evidence for the teacher version of the Child Symptom Inventory-4. School Psychology Quarterly, 19, 50-71.

Gal, E. (2006). An investigation of the relationship of sensory differences and intellectual level to stereotyped and self-injurious movements in children. Unpublished doctoral thesis, Curtin University of Technology, Perth, Western Australia.

Gal, E., Dyck, M., \& Passmore A. (2002). Sensory differences and stereotyped 
movements in children with autism. Behaviour Change, 19, 207-219.

Gal, E., Dyck, M., \& Passmore A. (2009). The relationship between stereotyped movements and self-injurious behavior in children with developmental or sensory disabilities. Research in Developmental Disabilities, 30, 342-352.

Gal, E., Dyck, M., \& Passmore, A. (in press). Relationships between stereotyped movements and sensory processing disorders in children with and without developmental or sensory disorders. American Journal of Occupational Therapy.

Gomez, R. (2008). Item response theory analyses of the parent and teacher ratings of the DSM-IV ADHD Rating Scale. Journal of Abnormal Child Psychology, 36, 865-885.

Gosch, A., Brambring, M., Gennat, H., \& Rohlmann, A. (1997). Longitudinal study of neuropsychological outcome in blind extremely low-birthweight children. Developmental Medicine \& Child Neurology, 39, 297-304.

Hamers, J. H. M., Hessels, M. G. P., \& Pennings, A. H. (1996). Learning potential in ethnic minority children. European Journal of Psychological Assessment, 12, 183-192.

Jan, J. E., Freeman, R. D., \& Scott, E. P. (1977). Visual impairment in children and adolescents. New York: Grune \& Stratton.

Jan, J. E., Groenveld, M., \& Connolly, M. B. (1990). Head shaking by visually impaired children: A voluntary neurovisual adaptation which can be confused with spasmus nutans. Developmental Medicine \& Child Neurology, 32, 1061-1066.

Koomar, J. A., \& Bundy, A. C. (2002). Creating direct intervention from theory. In A. C. Bundy, S. J. Lane, \& E. A. Murray (Eds.), Sensory integration: Theory and practice (2nd ed., pp. 261-302). Philadelphia: F. A. Davis.

Kurtz, P. F., Chin, M. D., Huete, J. M., Tarbox, R. S. F., O'Connor, J. T., Paclawskyj, T. R., \& Rush, K. S. (2003). Functional analysis and treatment of self-injurious behavior in young children: A summary of 30 cases. Journal of Applied Behavior Analysis, 36, 205-219.
Mangeot, S. D., Miller, L. J., McIntosh, D. N., McGrath-Clarke, J., Simon, J., Hagerman, R. J., \& Goldson, E. (2001). Sensory modulation dysfunction in children with attention-deficit-hyperactivity disorder. Developmental Medicine \& Child Neurology, 43, 399-406.

Mason, G. J. (1991). Stereotypes: A critical review. Animal Behavior, 41, 1015-1037.

Massa, J., Gomes, H., Tartter, V., Wolfson, V., \& Halperin, J. M. (2008). Concordance rates between parent and teacher clinical evaluation of Language Fundamentals $\mathrm{Ob}$ servational Rating Scale. International Journal of Language \& Communication Disorders, 43, 99-110.

McHugh, E., \& Lieberman, L. (2003). The impact of developmental factors on stereotypic rocking in children with visual impairments. Journal of Visual Impairment \& Blindness, 97, 453-474.

McHugh, E., \& Pyfer, J. (1999). The development of rocking among children who are blind. Journal of Visual Impairment \& Blindness, 93, 82-95.

McIntosh, D. N., Miller, L. J., Shyu, V., \& Dunn, W. (1999). Short Sensory Profile. San Antonio, TX: Psychological Corporation.

Miller, L. J., Anzalone, M. E., Lane, S., Cermak, S. A., \& Osten, B. (2007). Concept evolution in sensory integration: A proposed nosology for diagnosis. American Journal of Occupational Therapy, 61, 135-140.

Miller, L. J., \& Lane, S. J. (2000). Toward a consensus in terminology in sensory integrative theory and practice: Part 1: Taxonomy of neurophysiological processes. Sensory Integration Special Interest Section Quarterly, 23, 1-4.

Miller, L. J., Lane, S. J., Cermak, S. A., Anzalone, M., \& Osten, B. (2005). Regulatory-sensory processing disorders in children. In S. I. Greenspan \& S. Wieder (Eds.), Diagnostic manual for infancy and early childhood: Mental health, developmental, regulatory-sensory processing and language disorders and learning challenges (ICDL-DMIC) (pp. 73-112). Bethesda, MD: Interdisciplinary Council on Developmental and Learning Disorders. 
Miller, L. J., Reisman, J. E., McIntosh, D. N., \& Simon, J. (2001). An ecological model of sensory modulation: Performance of children with fragile $\mathrm{X}$ syndrome, autistic disorder, attention-deficit/hyperactivity disorder, and sensory modulation dysfunction. In S. S. Roley, E. I. Blanche, \& R. C. Schaff (Eds.), Understanding the nature of sensory integration with diverse populations (pp. 57-88). Tucson, AZ: Therapy Skill Builders.

Murdoch, H. (1996). Stereotyped behaviors in deaf and hard of hearing children. American Annals of the Deaf, 141, 379-386.

O'Donnell, L. M., \& Livingston, R. L. (1991). Active exploration of the environment by young children with low vision: A review of the literature. Journal of Visual Impairment \& Blindness, 85, 287-291.

Poustka, F., \& Lisch, S. (1993). Autistic behavior domains and their relation to selfinjurious behavior. Acta Paedopsychiatry, $56,69-73$.

Rogers, S. J., Hepburn, S. L., \& Wehner, E. (2003). Parent reports of sensory symptoms in toddlers with autism and those with other developmental disorders. Journal of Autism \& Developmental Disorders, 33, 631-642.

Rojahn, J., \& Sisson, A. L. (1990). Stereotyped behavior. In J. L. Matson (Ed.), Handbook of behavior modification with the mentally retarded (2nd ed., pp. 181225). New York: Plenum Press.

Schopler, E. (1995). Parent survival manual. New York: Plenum Press.

Smith, I. M., \& Bryson, S. E. (1994). Imitation and action in autism: A critical review. Psychological Bulletin, 116, 259-273.

Tröster, H., \& Brambring, M. (1993). Early motor development in blind infants. Journal of Applied Psychology, 14, 83-106.

Tröster, H., Brambring, M., \& Beelmann, A. (1991). Prevalence and situational causes of stereotyped behaviors in blind infants and preschoolers. Journal of Abnormal Child Psychology, 19, 569-590.

Turner, M. A. (1999). Annotation: Repetitive behavior in autism: A review of psychological research. Journal of Child Psychology \& Psychiatry, 40, 839-849.

Wehmeyer, M. L. (1989). Repetitive motor behaviors in young children at risk for severe developmental delay: Implications for pathological stereotypes in mental retardation. Unpublished Ph.D. dissertation, University of Texas, Dallas.

Eynat Gal, Ph.D., lecturer, Occupational Therapy Department, Room 911, Eshkol Building, University of Haifa, Mount Carmel, Haifa, 31905, Israel; e-mail: <egal@univ.haifa.ac.il>. Murray J. Dyck, Ph.D., professor, School of Psychology, Griffith University, Gold Coast, Queensland, Australia, QLD 4222; e-mail: <m.dyck@griffith. edu.au>. 